\title{
Althusser : une nouvelle pratique de la philosophie entre politique et idéologie. Conversation avec Étienne Balibar et Yves Duroux (Partie I)
}

Étienne Balibar, Yves Duroux, Fabio Bruschi et Eva Mancuso

\section{(Q) OpenEdition}

\section{Journals}

\section{Édition électronique}

URL : http://journals.openedition.org/grm/641

DOI : $10.4000 / \mathrm{grm} .641$

ISSN : $1775-3902$

\section{Éditeur}

Groupe de Recherches Matérialistes

\section{Référence électronique}

Étienne Balibar, Yves Duroux, Fabio Bruschi et Eva Mancuso, « Althusser : une nouvelle pratique de la philosophie entre politique et idéologie. Conversation avec Étienne Balibar et Yves Duroux (Partie I) », Cahiers du GRM [En ligne], 7 | 2015, mis en ligne le 07 juin 2015, consulté le 30 avril 2019. URL : http:// journals.openedition.org/grm/641; DOI : 10.4000/grm.641

Ce document a été généré automatiquement le 30 avril 2019.

(c) GRM - Association 


\title{
Althusser : une nouvelle pratique de la philosophie entre politique et idéologie. Conversation avec Étienne Balibar et Yves Duroux (Partie I)
}

\author{
Étienne Balibar, Yves Duroux, Fabio Bruschi et Eva Mancuso
}

\section{NOTE DE L'ÉDITEUR}

Nous publions ici la transcription d'une conversation avec Étienne Balibar et Yves Duroux que nous avons enregistrée le premier mars 2015 à Paris et à laquelle Andrea Cavazzini a également participé. Le point de départ de cette discussion est un texte que nous avions envoyé à $\mathrm{E}$. Balibar et $\mathrm{Y}$. Duroux afin de les interpeller et d'amorcer le dialogue. Dans la mesure où il ne contenait pas vraiment des questions ponctuelles, mais plutôt un questionnement visant à traverser la trajectoire d'Althusser à partir de quelques-unes de ses problématiques fondamentales, E. Balibar et Y. Duroux ont proposé des « variations libres » sur les thématiques que nous leur avions suggérées. Cet entretien se compose donc d'une part des questions que nous avions envoyées à nos interlocuteurs et d'autre part de la retranscription de la conversation qui en a découlé. Pour des raisons pratiques, nous avons divisé le dialogue en deux parties : l'une publiée dans ce numéro et l'autre dans le $8^{e}$ numéro des Cahiers du GRM consacré lui aussi à la pensée d'Althusser.

\section{Questions}

\section{Althusser et la politique}

1 La première série de questions que nous aimerions vous poser porte sur la conception althussérienne de la pratique politique. Bien qu'au premier regard ce thème puisse 
paraître secondaire dans l'œuvre d'Althusser, force est de constater que, dès ses premiers essais, tout particulièrement "Contradiction et surdétermination", c'est bien la construction d'une pensée de et pour la pratique politique qui constitue le souci central de la réflexion althussérienne.

\section{Sur la différence et le rapport structure/conjoncture}

2 Cette réflexion sur la politique semble d'emblée, et de manière constante tout au long de l'œuvre d'Althusser, devoir passer par la détermination du rapport entre structure et conjoncture. Ce n'est donc pas par hasard qu'il s'agisse d'un des problèmes les plus débattus de la pensée d'Althusser. Même parmi les althussériens, ce couple conceptuel a été d'emblée perçu comme marquant une distinction, voire même une opposition, d'où le fait que, comme l'écrit Balibar dans «L'objet d'Althusser " ${ }^{1}$, il serait possible d'identifier des althussériens de la structure et des althussériens de la conjoncture. À la lumière des inédits publiés dans les dernières années, on peut toutefois remarquer qu'Althusser a de manière assez cohérente toujours considéré ces deux termes comme deux spécifications d'un même objet, qu'on pourrait définir à partir des textes des années 80 comme le devenir-nécessaire de la contingence. Peut-on considérer que ce problème hantait Althusser dès le début des années 60 ? Est-ce que de ce point de vue Lire "Le Capital " diffère de Pour Marx, et ces deux ouvrages des écrits des années 80 ? Quel est le sens de l'identification tendancielle de la structure et de la conjoncture et pourquoi fallait-il néanmoins marquer leur différence? De quelle manière la détermination du rapport entre structure et conjoncture permet-elle de penser la pratique politique et peut-elle être mise au service de celle-ci? Cette question nous parait d'autant plus importante qu'elle permet de saisir la place et l'importance changeante de la lutte des classes dans la pensée d'Althusser. D'autre part, dans "L'objet d'Althusser ", avant de faire allusion à la distinction entre les althussériens de la conjoncture et ceux de la structure, Balibar suggère que la critique du déterminisme du sens de l'histoire «au nom de la singularité des conjonctures » constitue le « côté léniniste et plus encore machiavélien » des analyses d'Althusser. Pourriez-vous revenir sur la place de Machiavel dans le dispositif althussérien et dans ses transformations? Quel rôle a joué la lecture de Gramsci dans le rapport d'Althusser à Machiavel dans les années 1960 et puis dans les années 1970 ?

\section{Sur le communisme}

3 La présence de Machiavel devient ainsi de plus en plus significative chez Althusser à partir des années 70. Elle lui permet entre autres de revenir sur la question de la pratique politique par le biais d'une réflexion sur les rapports entre fins et moyens. Cette question nous donne accès à une problématique (rarement analysée par les lecteurs d'Althusser) qui nous paraît tout à fait essentielle, à savoir le rapport entre Althusser et ce que certains appelleraient aujourd'hui l'« Idée du communisme ». Althusser a toujours veillé à ne pas se lancer dans des spéculations sur ce que pourrait être la société communiste à venir, sans doute pour esquiver ces formes d'utopie qui pourraient entraver «l'analyse concrète de la situation concrète ». Il s'est limité tout au plus à parler d'«îlots de communisme " supposément déjà présents dans les interstices de notre société. Il nous semble néanmoins que quelque chose comme une idée du communisme soit à l'œuvre dans sa pensée, notamment lorsqu'il s'efforce de penser, de manière plus explicite qu'auparavant, la pratique politique communiste (mais peut-être aussi la pratique du 
philosophe matérialiste). C'est ce qui ressort tout particulièrement des débats des années 70 autour de la critique de l'État et de la dictature du prolétariat. Cela émerge clairement dans l'ouvrage de Balibar Sur la dictature du prolétariat ${ }^{2}$. Dans cet ouvrage, la différence entre, d'un côté, la dictature du prolétariat, et de l'autre, la gestion «bourgeoise » de l'État et des pays du socialisme réel est marquée par l'idée que dans la dictature du prolétariat la politique devrait être réfléchie du point de vue du communisme, ce qui rend impossible toute réduction de la politique à un mode de gouvernement. Il nous semble qu'au fond Althusser pensait quelque chose de similaire lorsqu'il réfléchissait à cette nouvelle pratique politique que devrait être la politique communiste (ainsi qu'à cette nouvelle pratique de la philosophie que devrait être la philosophie matérialiste), en parlant par exemple de "stratégie communiste ", et ceci malgré vos différends sur la question de savoir si cette nouvelle pratique de la politique devait avoir lieu hors État ou au sein de l'État. Pourriez-vous revenir sur ce débat en nous aidant à mieux comprendre le rôle éventuel de quelque chose comme une idée du communisme chez Althusser?

\section{Althusser et le subjectif}

4 La question de la pratique (politique ou autre) se rattache systématiquement chez Althusser à celle du sujet - catégorie qu'il reléguait dans la sphère de l'idéologie. Si l'on connait la conception althussérienne de l'idéologie, on ne peut toutefois pas penser que le but d'Althusser était par-là de faire disparaître purement et simplement cette catégorie. Pour marquer la spécificité de la position d'Althusser à propos de la catégorie de sujet, Badiou affirme ainsi que, si chez Althusser il n'y a pas de théorie du sujet, il y a bien quelque chose comme une théorie du «subjectif sans sujet $»^{3}$. La question est ainsi de savoir si et comment Althusser a été capable d'en saisir la nature et la fonction spécifique.

\section{Sur la science et l'idéologie}

5 Le premier problème qui se pose à ce niveau est bien connu. Althusser oscille entre l'idée que la science peut transformer « de l'extérieur » l'idéologie lorsqu'elle est transmise aux masses par la formation théorique et l'idée qu'elle doit être elle-même "idéologisée " pour être efficace, qu'elle doit donc être doublement inscrite dans la topique - ce qui suppose que quelque chose doit se passer au sein même de l'idéologie pour que la science puisse y intervenir efficacement (on peut penser aux idées esquissées par Althusser d'une pluralité d'interpellations ou d'une interpellation qui se décentre au moment même où elle est lancée). Cette question recoupe ainsi le problème du «théoricisme» et du "pédagogisme " d'Althusser. En revenant plus généralement sur les enjeux de l'autocritique d'Althusser, pourriez-vous nous dire si et comment les transformations de la position d'Althusser concernant la pratique théorique ont correspondu à une transformation du statut de la catégorie de sujet? L'enjeu principal de ces transformations est par ailleurs la conception althussérienne de la philosophie. Celle-ci semble ainsi devoir jouer un rôle majeur dans la détermination de la conception althussérienne du subjectif. En même temps, s'il n'y a pas de sujet de la science et que le sujet est toujours idéologique, peut-on parler de quelque chose comme un sujet de la philosophie ? À la lumière de ces remarques, est-ce que, à votre avis, la célèbre critique de Rancière à l'égard d'Althusser a visé la bonne cible et a été capable de l'atteindre ? 


\section{Sur Spinoza}

6 La question du sujet pourrait par ailleurs être articulée à celles des affects et de l'inconscient. Dans les chapitres de L'avenir dure longtemps consacrés à Spinoza et Machiavel, Althusser traite du « déplacement interne des "passions tristes" en "passions joyeuses" $»^{4}$ que l'on trouve chez Spinoza et qui «fait tout à fait penser par avance à Freud ». Dans ses écrits autocritiques, Althusser a rattaché sa conception de la coupure de la science avec l'idéologie à la théorie spinoziste du passage du premier au deuxième genre de connaissance. Cependant, rien n'y est dit sur la question de la "transition éthique ", c'est-à-dire de la conversion des passions tristes en passions joyeuses. Et, même si l'intérêt d'Althusser pour la psychanalyse et la question du déplacement semble transparaitre dans "Le "Piccolo", Bertolazzi et Brecht " ${ }^{5}$, où il parle d'un rapport actif à l'idéologie qui pourrait peut-être renvoyer à la transition éthique de Spinoza, la coupure n'est jamais, dans Pour Marx, explicitement définie comme cette conversion passionnelle cette transformation subjective - que chacun pourrait opérer en droit. À partir de là, la rupture avec l'idéologie nécessite-t-elle obligatoirement, pour Althusser, un passage par la connaissance scientifique ? La " coupure » est-elle seulement rupture avec un système conceptuel déterminé, ou bien peut-elle également être une prise de distance avec certaines déterminations de l'idéologique défini comme cette «structure imaginaire qui existe non seulement sous la forme de concepts, mais aussi sous la forme d'attitudes, de gestes, de conduites, d'intentions, d'aspirations, de refus, de permissions, d'interdits, etc. $»^{6}$, prise de distance qui à la fois requiert et rend possible un certain déplacement passionnel ? À l'époque de Pour Marx, Althusser appréhende-t-il déjà Spinoza à partir de cette question de la conversion des passions, lit-il Spinoza à travers Freud afin de penser la question de la prise de distance avec l'idéologie ? Et s'il ne le fait pas, y a-t-il des raisons à cela? Celles-ci ont-elles un lien avec le problème posé par le rapport des intellectuels aux masses? D'autre part, quel est le rapport d'Althusser à l'ouvrage de Deleuze sur Spinoza lorsqu'il sort en $1969^{7}$ ? L'a-t-il lu ? Et si c'est le cas, est-il possible, selon vous, que cette lecture ait eu une quelconque incidence sur son rapport à Spinoza?

\section{Sur la psychanalyse}

7 Le problème de la conversion passionnelle implique d'aborder le rapport d'Althusser à la psychanalyse. Nous aimerions d'abord vous demander de nous parler du «groupe Spinoza ", où l'étude du rapport entre idéologie et inconscient, et la question de l'effetsujet, occupent une place centrale. Le travail de ce groupe nous semble à certains égards constituer la version proprement althussérienne des Cahiers pour l'analyse (auxquels ont d'ailleurs collaboré plusieurs membres du groupe). Quel rôle vouliez-vous faire jouer au rapport entre marxisme et psychanalyse et par conséquent entre idéologie et inconscient? Plus tard, dans L'avenir dure longtemps, Althusser pense la virtù machiavélienne à partir de concepts freudiens (inconscient, déplacement, etc.) pour faire du Prince une sorte d'analyste. Cette façon d'appréhender Machiavel à travers la psychanalyse était-elle déjà présente dans les années 1960 ? Ou bien, est-ce que le point de contact entre Althusser et la psychanalyse change après la tentative du groupe Spinoza? On a en effet l'impression qu'Althusser abandonne de plus en plus toute tentative d'articuler directement (disons au niveau de leurs objets) marxisme et psychanalyse et que c'est justement une analyse des rapports entre le geste du philosophe 
matérialiste et de l'analyste qui prend sa place. Dans quelle mesure peut-on trouver chez Althusser une conception « analytique » de l'intervention intellectuelle?

\section{Conversation avec Étienne Balibar et Yves Duroux (Partie I)}

8 Yves Duroux : Il faut dire que vous ne proposez pas simplement des questions, mais une sorte de reconstruction d'une trajectoire d'Althusser, et les deux thématiques, la politique et le sujet, sont en continuité, il n'y a pas de rupture. Ça commence par des choses très classiques - le rapport structure/conjoncture. Mais on voit que ce qui vous intéresse, derrière ces choses très classiques, c'est ce qu'on peut en tirer, au-delà d'une opposition qui serait simplement conceptuelle, concernant ce passage par la politique qui se termine sur la psychanalyse. Il ne s'agit pas vraiment de questions ; je dirais plutôt que c'est un questionnement.

Étienne Balibar : Commençons par le communisme, sur Lire «Le Capital »" on y reviendra... Évidemment vous savez que je suis l'un des coauteurs de Lire "Le Capital », mais Lire "Le Capital» c'était un séminaire, c'était un groupe de travail qui s'était mis en place avant que le séminaire commence. C'était un projet qui avait mûri progressivement; on y pensait depuis longtemps et naturellement on était plusieurs là-dedans, y compris des gens qui n'ont pas fait des exposés oraux en forme, mais sans qui rien de tout ça n'aurait eu lieu, et en particulier Yves. Il en sait donc autant et peut-être même plus que moi sur Lire "Le Capital», parce que moi, à un moment donné, je me suis embarqué dans une direction, en partie suggérée par Althusser et en partie choisie par moi - la même chose est vraie pour Rancière. Pour Macherey et Establet, ce sont deux cas un peu différents : ils n'étaient plus élèves à l'ENS en 64-65 (mais naturellement ils avaient été très actifs dans le groupe althussérien les années précédentes). Une des choses que je crois de plus en plus et que, comme c'est l'année de l'anniversaire de Lire «Le Capital », j'ai essayé de dire sous une forme aussi juste que possible, c'est que non seulement Lire «Le Capital» était un projet collectif auquel Althusser nous a associés, mais que certaines de ses spécificités, y compris dans les propres textes d'Althusser - et malgré le fait qu'évidemment il avait des idées derrière la tête -, relèvent du fait qu'Althusser était obligé pour son propre compte de ressaisir de son point de vue à lui quelque chose qui avait été fait en collaboration et dont il n'était pas entièrement le maitre. D'ailleurs, Rancière a très bien expliqué, dans les interviews récentes ${ }^{9}$, qu'Althusser a eu cette idée extraordinaire de nous dire: "Le marxisme est à refonder, mais il faut qu'on le fasse ensemble ; j'ai besoin de vous pour le faire, etc.». C'était extraordinairement excitant parce qu'il ne nous disait pas: "Apprenez le marxisme ». On avait 20-25 ans et il nous disait : «Faisons-le, je compte sur vous pour trouver ce qu'il faut dire ». Là il faut tenir compte du fait qu'il y a une chronologie, qui suit l'ordre inverse de l'ordre de la publication: la préface a été écrite après-coup, donc c'est une sorte de réflexion et en même temps d'une certaine façon une récupération. C'est pourquoi je pense que le contenu de Lire «Le Capital» n'est pas de l'althussérisme pur - les divergences possibles avec Pour Marx ${ }^{10}$, par exemple sur la question structure/conjoncture, se situent en partie là-dedans. Ce qui revient à dire que nous avons injecté quelque chose là-dedans, qui n'allait pas contre ses intentions, mais qui les tordait dans un sens "épistémologique ", pour le dire vite, en ce qui concerne Yves et moi, et dans une moindre mesure Rancière dont la formation philosophique était un peu différente. C'est pourquoi, aujourd'hui, je suis à la fois gêné et amusé d'avoir à 
parler de Lire «Le Capital » parce qu'une des choses que je veux dire c'est qu'au fond je ne savais pas grand-chose. J'étais un philosophe apprenti et évidemment j'avais une grande capacité d'imitation, mais d'une certaine façon la philosophie qu'il y a dans Lire "Le Capital » n'est pas purement celle d'Althusser.

YD : Étienne a parfaitement raison : entre l'exposé ${ }^{11} \mathrm{~d}^{\prime}$ Althusser et la préface ${ }^{12}$, il y a une grande différence. Il m'avait envoyé sa préface et puis un jour il me téléphone et me dit: «Il faut que tu me le rendes", parce qu'il n'en avait qu'un exemplaire. Je lui dis : "Je rentre à Paris demain et je te le ramène ». Et là il m'a demandé ce que j'en pensais. Alors je dis : « Là tu es allé quand même très loin dans le structuralisme ». Parce qu'il y avait un texte ronéotypé auquel j'avais travaillé avec Miller et Milner, qui date de fin 64 et qui avait circulé dans l'Ecole : «Action de la structure $»^{13}$. Althusser l'avait lu, Rancière l'avait lu, etc. Et ce texte est un texte étrange - j'expliquais ça dans un entretien en anglais ${ }^{14}$. Althusser m'a dit : «Oui t'as raison, je vais faire une préface un peu différente ». Et c'est vrai que le ton de la préface est différent. Mais paradoxalement l'effet de Lire "Le Capital » a moins été produit par la préface, que par le texte d'Étienne, extrêmement important parce que c'est un texte très pédagogique. Immédiatement après, fin 65 , il y a eu ce qu'on appelait des cours de formation théorique, auxquels Rancière a participé (nous on ne pouvait plus, on était partis), et ce qui a été enseigné c'était le texte d'Étienne "Le concepts fondamentaux du matérialisme historique ».

11 EB : Du coup Rancière a été obligé d'enseigner mon texte ! C'est parce que c'est celui qui constituait une réécriture (c'était toujours l'une des obsessions d'Althusser, il l'a dit : «Je veux écrire des manuels ») du canon marxiste-léniniste, en fait stalinien, du matérialisme historique. exemple, le texte de Rancière, c'est moi qui lui ai conseillé le titre ${ }^{15}$. Je lui ai dit : «Comme tu as fait ton diplôme sur le jeune Marx, tu nous expliqueras pourquoi le vieux Marx n'est plus le jeune Marx ». Il a fait ça très bien d'une certaine façon, en posant quelques questions auxquelles personne n'a jamais répondu. À un moment donné, il dit: «Pourquoi Marx affirme que les économistes classiques, ce ne sont pas les économistes vulgaires?» Mais néanmoins l'articulation de son texte suit l'idée qu'on passe de l'idéologie du jeune Marx à la science du Marx mûr. En ce qui concerne les deux autres textes: celui d'Establet ${ }^{16}$, c'est un texte rédigé après-coup. Establet a lu les textes déjà existants et il a conçu quelque chose après-coup sur le plan du Capital. Et celui de Pierre Macherey ${ }^{17}$, c'est quelque chose que j'appellerais micrologique : il a fait un exposé détaillé sur trois pages du début du Capital pour expérimenter une méthode de lecture... Mais, c'est vrai qu'il faut bien dire que l'effet de Lire "Le Capital», texte composite, disons déséquilibré et contradictoire, a été produit malgré tout par le texte d'Étienne et celui d'Althusser.

EB : Du coup, ça conduit à la question de savoir ce qu'on pense du texte central d'Althusser. Dans le petit texte que je vous ai envoyé ${ }^{18}$, j'y suis allé au marteau, à l'emporte-pièce. Je dis qu'en relisant le texte d'Althusser, il m'a semblé qu'il y avait des choses très fortes philosophiquement, très intéressantes, et j'ai mentionné le temps 
historique et la lecture symptomale. Pour rester au texte central, il y a la question du temps historique. Aujourd'hui on lit ça d'un œil différent parce qu'il y a eu tellement de choses sur cette question de la multiplicité, de la complexité, du temps. Puis les gens ont lu Benjamin ; ils ont un peu moins lu Ernst Bloch, en quoi ils ont tort parce que je pense que c'est une des comparaisons les plus intéressantes. Mais Althusser ne connaissait absolument pas ça et s'il l'avait connu il aurait été contre évidemment. Il travaillait avec l'historiographie française des Annales, la sociologie française, Halbwachs, pour essayer de fabriquer une conception objective de la multiplicité du temps historique. Et puis il travaillait dans le voisinage immédiat - c'est une chose qui me frappe après-coup - de Derrida qui faisait un cours sur historicité et temporalité chez Heidegger dans la salle d'à côté, que nous on n'a pas boycotté au sens actif du terme, mais où on n'est pas allés. Mais, Althusser lui il était surement au courant. Ils devaient en parler entre eux. Les traces heideggériennes ou les allusions qui se trouvent dans le texte ne sont donc sûrement pas là par hasard. Ça faisait partie de leur fond commun et c'étaient des choses dont il discutait avec Derrida. J'ai dit que c'est philosophiquement très fort. Ensuite il y a des choses qui sont scolaires, comme les passages sur l'objet de l'économie politique. Je ne range pas dans les choses scolaires sa grande idée épistémologique : la mise en œuvre de l'idée de la coupure, la lecture du texte d'Engels, etc. C'était Bachelard qui tout d'un coup trouvait à s'employer. Et puis, il y a quelque chose que j'ai relu avec un effarement absolu: c'est le célèbre chapitre, qui a eu une énorme influence pour des raisons politiques : «Le marxisme n'est pas un historicisme ». Un exemple parfait de la méthode stalinienne de critique des déviations de gauche (Lukacs) et de droite (Gramsci) fondée sur l'idée que leur fond commun est le même, c'est-à-dire l'ignorance du caractère scientifique du matérialisme historique. C'est un texte absolument effarant. Évidemment, Althusser a écrit d'autres choses ensuite sur Gramsci. Vittorio Morfino vient de faire un très bon travail pour essayer de mettre un peu à plat les différents moments ${ }^{19}$. Mais ça exprime aussi le cœur des intentions politiques d'Althusser à l'époque de Lire "Le Capital " , dont il faut qu'on parle en relation à son rapport au communisme.

YD : Étienne, est-ce que je peux te poser une question? Sortent en même temps, fin 65, Lire «Le Capital» et Pour Marx. Ces deux livres ne sont pas du tout pareils. Qu'est-ce qui a eu plus d'influence? Parce que moi j'ai presque l'impression, je suis obligé de le dire, que c'est Lire «Le Capital». C'est pourquoi Althusser a fait cette sorte de réaction violente après-coup (déjà dans l'avertissement à la réédition il a dit que le texte était affecté par " une erreur dans la conception même »). Et pourquoi c'est Lire «Le Capital»? Alors ça a été relayé par le fameux manuel de Marta Harnecker ${ }^{20}$.

EB : Beaucoup plus tard... Avant d'être relayé par Marta Hanecker, il a été relayé par le texte qu'Althusser avait publié dans les Cahiers marxistes-léninistes, qui avait été traduit en espagnol à Cuba, sur «Matérialisme historique et matérialisme dialectique $»^{21}$. On est en plein dans la réécriture de Staline, une fois de plus.

YD : Oui, bon. C'est pour ça je pense que cette question est importante, parce que votre question porte sur la politique. Mais c'est très important de dire qu'en 65 dans Lire «Le Capital» il y a quelque chose qui s'est mis en place sur une certaine conception de la politique. Tu vois bien que la «Lettre à Krasucki » ${ }^{22}$ est complètement homogène à Lire "Le Capital». Althusser allait former un bataillon de jeunes théoriciens, qui allaient expliquer au Parti ce que c'est que la science - c'était quand même ça -, pour remettre le Parti sur ses pieds. 
19 EB : Ça va nous mener à discuter de l'idée qu'Althusser se faisait du PC, du rôle de la théorie dans le Parti, et donc de son propre rôle. Toutes choses dont il faut essayer de parler.

20 Fabio Bruschi : Mais c'est vrai qu'avec cette question sur structure/conjoncture, on voulait en venir à la manière dont, aux yeux d'Althusser, ces problèmes pouvaient avoir une retombée politique. Ce que vous venez dire sur le fait qu'Althusser a dû rattraper après-coup ce qu'il avait fait commencer...

21 EB : C'est plusieurs choses à la fois. En effet on peut dire qu'il a fallu qu'il rattrape et, en rattrapant, il a renoué avec des choses qu'il avait lui-même écrites antérieurement et qui s'étaient un peu perdues dans le projet de Lire «Le Capital». Mais d'un autre côté il le faisait dans une nouvelle conjoncture au milieu des bagarres avec les maos d'un côté et le Parti de l'autre, soumis à l'accusation d'avoir oublié la lutte de classes, à laquelle à mon avis il a été exagérément sensible, mais on comprend pourquoi. Et, du coup, il n'a pas simplement essayé de rectifier une déviation, mais de fabriquer encore une autre version de son discours, de sa théorie - chose d'ailleurs qu'il n'a jamais cessé de faire tout au long de sa vie. Ça ne veut pas dire qu'il n'y a pas des constantes.

Pour revenir à structure/conjoncture, vous vous référez à ce que j'ai écrit là-dessus et vous avez raison quand vous dites que, pour lui, les deux problèmes ne sont pas différents. C'est moi qui ai dit plus tard qu'il me semblait qu'il y avait deux tendances chez Althusser, mais peut-être j'ai au fond une fois de plus vu les choses à travers mes propres lunettes, c'est-à-dire que comme j'avais le sentiment d'avoir produit dès le début une interprétation d'Althusser qui était plutôt structuraliste, dans tous les sens du mot, à la fois le sens philosophique français et le sens marxiste ou quasi-marxiste de l'analyse de la structure sociale, qui fait corps dans des textes célèbres de Marx avec des visions de philosophie de l'histoire où la conjoncture n'occupe aucune place - il y a de l'eschatologie, mais il n'y a pas de conjoncture -, comme j'avais le sentiment d'avoir tiré les choses de ce côté-là et que je comprenais bien qu'il y avait aussi un autre côté qui était tout aussi important pour Althusser ou même plus, j'ai fabriqué ce petit schéma d'interprétation qui consistait à lui attribuer ou à lire chez lui un écart constamment reproduit et en même temps constamment retravaillé pour le résorber. Mais il se peut que ça soit ma propre invention. D'ailleurs, ça ne veut pas dire que Marx lui-même ignorait la conjoncture ; il s'en occupait beaucoup par ailleurs, mais pas exactement dans les mêmes textes. J'ai dit qu'Althusser a fabriqué un Marx à la Montesquieu d'un côté et un Marx à la Machiavel de l'autre, parce que je pensais à son insistance dans la conclusion $\mathrm{du}$ Montesquieu $^{23}$ sur l'importance de la découverte par Montesquieu du continenthistoire, qu'il centrait autour de la transformation du sens de l'idée de rapport (évidemment le rapport social c'était notre schibboleth pour entrer dans le matérialisme historique), et puis de l'autre je pensais au Marx-Machiavel, c'est-à-dire au Marx-Lénine, en faisant un court-circuit très rapide. Alors, il n'y a pas de doute que Machiavel et Montesquieu ne sont pas la même chose, bien qu'ils soient des penseurs qui se situent hors du droit naturel ou encore ceux que Arendt, totalement ignorée d'Althusser, a classés parmi les non-philosophes ou les anti-philosophes - on pourrait presque dire dans le champ politique. Je ne dis donc pas qu'ils n'ont aucune affinité, mais leur objet n'est quand même pas exactement le même. C'est une petite grille, et elle a ses limites.

22 YD : Ça désigne quand même une sorte d'entrée à deux portes, dont on ne sait pas trop d'une certaine façon comment les faire tenir, parce que ce n'est pas par hasard si le premier livre d'Althusser porte sur Montesquieu et le dernier livre sur Machiavel. 
EB : J'ai tendance à penser - mais c'est en partie de l'imaginaire - qu'Althusser s'est trouvé lui aussi, à sa façon et à son époque, dans la même situation que tous les marxistes de l'histoire sans aucune exception à commencer par Marx lui-même. C'est des gens qui pensent l'histoire en effet sur ces deux registres à la fois, en essayant de résorber la distance, entre la nécessité ou le processus, d'un côté, et l'intervention et donc l'événement politique, l'action politique, de l'autre. Le marxisme n'est pas le seul, mais le marxisme est à un haut degré une pensée théorique qui a comme problème de tenir les deux à la fois. D'un côté, il ne se satisfait absolument pas de l'idée que l'événement politique ou l'action soient le produit de la liberté ou l'irruption du Messie dans l'histoire: il y a des forces, il y a des agents, il y a des conditions; " tout tient aux conditions ", disait toujours Althusser. Et puis, de l'autre côté, le marxisme tente une sécularisation matérialiste ou une transformation de ce qu'était devenue la philosophie de l'histoire de l'âge des Lumières et de l'époque de la Révolution française, et surtout de la sociologie, de la tradition sociologique telle qu'elle est fondée au début du XIXe siècle, c'est-à-dire de l'idée que les sociétés ont des structures, que ces structures sont contraignantes en matière de représentations, d'institutions, de comportements et qu'elles se transforment. Quelqu'un qui avait très bien compris ça en nous lisant et qui m'avait fait des compliments dont je lui reste éternellement reconnaissant, ce n'est pas un marxiste, enfin si, c'est un para-marxiste qui a eu beaucoup d'influence sur certains de nos camarades, qui a en tout cas aidé leur carrière : c'est Georges Balandier, qui avait écrit un livre sur l'anthropologie politique africaine et disait que j'avais très bien exposé que le problème est celui de la transformation des structures dans le temps. Mais la tragédie du marxisme depuis Marx lui-même jusqu'à Althusser compris, c'est que ce qui se passe sous leurs yeux et, j'allais dire, sous leurs pas n'est jamais ce qu'ils ont prévu, c'est toujours autre chose, c'est même toujours le contraire. Les marxistes sont des gens qui perdent pied en permanence dans l'histoire qu'ils veulent comprendre et dont ils veulent être les agents. Alors on pourrait dire qu'ils n'ont qu'à renoncer à cette ambition absurde de vouloir comprendre le cours de l'histoire dont ils sont eux-mêmes les acteurs. Moi je ne dis pas qu'il faut renoncer. Althusser le savait naturellement, en tout cas pour ses prédécesseurs, c'est pour ça que dans Pour Marx évidemment, dans un de ses moments d'exaltation, il avait dit que Lénine a résolu le problème, qu'il a été le contemporain et l'acteur conscient de l'histoire qu'il était en train de faire parce que, d'un côté, il avait les clés théoriques qu'il fallait - le marxisme les lui avait données - et, de l'autre, il avait, appelons ça le génie si on veut, enfin il n'était pas un contemplatif, il savait qu'il fallait forcer le cours des choses, dans leur propre sens naturellement. Évidemment Althusser n'aurait jamais pu, sauf peut-être vers la fin, en 78 , en venir à l'idée terriblement pessimiste que la conviction de Lénine d'effectuer, de cristalliser dans la conjoncture la nécessité historique constituait une folie que les Soviétiques et d'autres ont mis des décennies à payer jusqu'à ce que tout ça s'effondre comme un château de cartes.

YD : Tu es un peu pessimiste, mais enfin...

EB : On n'est pas obligés d'aller jusqu'au bout de ce pessimisme, mais pour ce qui est du décalage je pense que c'est un point important...

YD : C'est vrai. Je pense que quand tu regardes sur cette question les derniers textes d'Althusser, tu vois que finalement il supprime l'idée de la structure. Dans un de ses tout derniers textes ${ }^{24}$, il critique le Marx du Capital en expliquant que le grand moment du Capital, c'est le chapitre sur l'accumulation primitive, parce que le chapitre sur l'accumulation primitive ne porte pas sur la reproduction au sens structural, sur la 
causalité structurale. D'une certaine façon, il soumet la structure à la conjoncture. Il dit : «Il n'y a qu'une question, mais il faut trouver un autre concept ». Le matérialisme c'est justement ce qui est au-delà de cette opposition... dogmatiques très forts et puis des moments d'autocritique semi-dépressifs, pour repartir. C'est une succession de séquences. Il y a la séquence de Lire "Le Capital » où l'on voit qu'il y a déjà un recul entre son texte, le texte d'Étienne et la préface. Beaucoup plus tard, il y a ce dernier texte qui m'avait énormément frappé parce que tout à fait à la fin de mon travail sur Marx - je me suis arrêté en 77 - j'étais arrivé à peu près à la même chose, à savoir à l'idée qu'effectivement on ne peut pas distinguer entre structure et conjoncture. Je recherchais même dans le concept de rapport de production ce qui pouvait ne pas être distinct conceptuellement du travail sur l'accumulation primitive. Et donc je crois quand même... Vous voulez sauver Althusser d'une certaine façon, et je crois que sur ce point c'est vrai qu'il y a quand même des choses très anciennes - comme dans « Contradiction et surdétermination » - qu'on retrouve tout à fait à la fin. Mais il y a des séquences d'intervention qui, à mon avis, se sont mises pour ainsi dire sur l'écran de la philosophie, parce que fondamentalement il faut vous dire qu'Althusser n'a jamais fait avancer le «matérialisme historique ». Même la théorie des appareils idéologiques d'État, qui a eu un effet extraordinaire, met en avant une catégorie philosophique. Finalement donc Étienne ne doit pas être d'accord - je pense que c'est par la philosophie, par le travail qu'il a fait sur la philosophie, par les déplacements successifs qu'il a donné à sa catégorisation de la philosophie qu'on peut voir ce que j'appelle cette succession de séquences. 
EB : Oui, je suis assez d'accord avec ça. Je crois qu'on peut ajouter que chez Althusser - je suis d'accord sur les séquences - il y a des questions qui sont mises de côté et qui ressortent. Évidemment la forme sous laquelle elles ressortent est conditionnée par le milieu ambiant. Quand on lit les correspondances d'Althusser on voit bien - et si j'osais je dirais que je comprends parce que j'éprouve exactement la même chose - qu'il a constamment le sentiment qu'il faut intervenir dans une discussion contemporaine pour l'infléchir, que ce n'est pas lui qui choisit les termes. Et d'un autre coté il a aussi l'idée qu'il a un projet de longue haleine auquel il faudrait finir par donner forme. Alors les deux choses se télescopent - le texte sur les appareils idéologiques d'État ${ }^{26}$, c'est tout à fait ça. C'est l'après-coup de 68 , c'est la réponse aux accusations de stérilité du marxisme qui sont portées à droite et à gauche, mais c'est aussi la tentative de faire entrer dans le matérialisme historique, comme tu le dis, par le biais de la philosophie, ces questions dont Gramsci avait traité, et qu'Althusser aurait voulu poser autrement, sur la place des intellectuels, la fonction de la science, etc.

Et en même temps, il y a des trucs qui l'obsédaient, des objets historico-politicoépistémologiques, et, parmi les objets en question - ça a beaucoup à voir avec le fait qu'Althusser est français, un marxiste français du milieu du XXe siècle -, il y avait la fameuse question de la transition de la monarchie absolue à la bourgeoisie. Je pense beaucoup à ça en ce moment parce qu'on m'a demandé de jeter un coup d'œil sur les épreuves de publication du dernier cours de Foucault encore à publier. Ce n'est pas La société punitive ${ }^{27}$, mais celui qui vient juste avant qui s'appelle Théories et Institutions pénales , le cours que Foucault a fait au Collège de France en 71-72. De ce cours-là, on n'a pas d'enregistrement, donc ça ne va pas pouvoir être édité sous la forme d'un texte continu comme les autres. Mais on a les notes préparatoires de Foucault : ce n'est pas aussi bien qu'un texte, on voit qu'il prépare des documents, des citations, des phrases qu'il veut placer. Il suit d'ailleurs son plan, mais évidemment il fabrique le texte oralement, donc c'est un peu maigre. Mais ce qui existe est quand même tout à fait suffisant pour voir de quoi il s'agit. Or, les éditeurs voulaient que je vérifie les allusions à Althusser et que je leur dise s'ils ne s'étaient pas gourés. Je leur ai dit : « Vous n'avez pas compris à quel point ce texte est obsédé par Althusser, est une réponse à Althusser ou est un défi à Althusser, parce qu'évidemment vous ne connaissez pas son livre sur Montesquieu ». Le cours de Foucault est entièrement consacré à la genèse de ce qu'il appelle l'appareil répressif d'État à l'occasion de la révolte fiscale des va-nu-pieds qui a été réprimée par le chancelier de Louis XIII et de Richelieu, le chancelier Séguier, grande figure de l'histoire politique et légale française. La base de Foucault, c'est l'ouvrage d'un historien soviétique qui s'appelait Boris Porchnev ${ }^{28}$, qui entretemps avait été traduit en français, mais qu'Althusser avait lu antérieurement dans une traduction allemande - j'ai vérifié qu'elle était à la bibliothèque de l'ENS - et dont il avait tiré tout l'arrière-plan historique de son livre sur Montesquieu. Alors, l'article d'Althusser sur les appareils idéologiques d'État vient d'être publié ; c'était un événement, il faut bien le dire. Et autour de Foucault dans le petit milieu interstitiel entre Foucault, nous, les maoïstes, Althusser, etc., le fait qu'Althusser publie un texte, même partiel et surtout peut-être sous cette forme énigmatique, c'était un événement et ça représentait un défi. Les uns, les althussériens professionnels, moi, Terray, Lecourt, etc. on est tout de suite montés au créneau, on a dit: "C'est génial, très important, c'est le point de départ de la théorie ", etc. Du côté des maos, du côté de Foucault, qui n'est pas la même chose que les maos, mais qui a des rapports avec eux, la question se pose de savoir comment on répond à ça. Et je pense que Foucault se dit - je ne dis pas ça contre lui - que le moment est venu de détacher les 
jeunes marxistes français d'une part d'Althusser et d'autre part, à travers Althusser, aussi du marxisme. C'était compliqué parce que Foucault ne voulait pas se débarrasser du marxisme de l'extérieur, sans mettre quelque chose à la place. Il voulait mettre quelque chose à la place, à partir du marxisme lui-même, ça se voit très bien dans La société punitive. Alors, Althusser a dit : «Ce qui manque au marxisme, c'est une théorie de l'État, parce que la théorie de l'État comporte deux moitiés : d'un côté les appareils répressifs d'État - dont on sait ce que c'est car Lénine et Marx lui-même nous l'ont très bien expliqué : c'est l'instrument dont la bourgeoisie se sert, etc. -, et de l'autre côté les appareils idéologiques d'État - dont le marxisme n'a rien compris, sauf Gramsci, qui a essayé un peu de faire quelque chose sur ce terrain, mais qui n'a pas eu des bons concepts -, donc moi je vais l'expliquer ». Foucault lui dit : « Ta-ra-ta-ta, l'appareil répressif d'État, c'est bien plus compliqué que ce que vous croyez; il n'y a pas du tout de théorie de l'appareil répressif d'État ni de son histoire, donc je vais la faire ». Mais il la fait avec les matériaux mêmes qu'Althusser avait déjà utilisés. Autrement dit, il plonge dans l'histoire de l'aspect politique de l'accumulation primitive et cela était l'obsession d'Althusser : la restitution à partir de quelques indications de Marx et d'autres éléments de la dimension politique de l'accumulation primitive. Donc quand il revient à cela à la fin, c'est une fois de plus une façon de ressortir une vieille rumination.

FB : Quand je disais qu'il a peut-être une continuité, je pensais justement à la thématique de l'accumulation primitive en général, telle que vous l'abordez par exemple dans votre texte dans Lire «Le Capital».

YD : Oui là je suis d'accord avec ce que tu dis, parce que toi tu vas le chercher encore plus loin, dans Porchnev, Montesquieu... Bon et si à partir de là on passait à Machiavel ? Moi la seule chose que je peux dire est que j'ai ses notes de cours, d'avant 60. Il y avait des choses sur le couple fortune/virtù et il expliquait que c'était totalement non-hégélien, il parlait de l'anti-hégélianisme du couple fortune/virtù. Donc d'une certaine façon il y a quelque chose qu'il travaillait déjà à partir de ça, mais je n'en sais rien de plus. Parce que le texte Machiavel et nous ${ }^{29}$ ''est dix ans après...

EB : C'est très compliqué l'histoire du texte sur Machiavel...

YD : Il me l'a donné son cours Althusser. Je me rappelle très bien. Il m'envoie un jour un gros paquet comme ça...

EB : Tu veux dire le livre Machiavel et nous? Ah oui, je t'en veux toujours mortellement. J'en veux d'abord à Althusser, qui a écrit un livre sur Machiavel sans jamais m'en avoir parlé et a fortiori le fait qu'il te l'ait montré, ça me fait bouillir de rage...

YD : Je me rappellerai toujours que dans la note d'accompagnement, Althusser m'écrit : "Yves, j'ai brulé mes vaisseaux ».

41 EB : Je crois que l'interprétation d'Althusser était pour l'essentiel en place ou en tout cas en route avant que Lefort ne publie son livre. Alors, il devait peut-être quelque chose à Merleau-Ponty, à la fois une incitation et une rivalité et même une formulation ou deux. Mais quand Althusser a lu le livre de Lefort, il a dû penser à la fois que c'était un travail considérable, il a dû être extrêmement admiratif, je n'ai pas de raisons d'en douter ; et en même temps, il a dû se dire que ça ne l'empêchait pas de faire ce qu'il était en train de faire.

Mais je voudrais ajouter quelque chose, pour faire le lien avec le moment précédent sur Machiavel et Montesquieu, sur la fortune et la virtù. Il y a quelque chose qui m'a souvent traversé l'esprit, et d'ailleurs j'avais eu des discussions avec Althusser à ce propos : c'est 
qu'il y a un schéma très simple, purement formel, qui joue un rôle très important dans la pensée d'Althusser. On pourrait l'appeler de différentes façons. Il joue un rôle à la fois dans ses lectures des textes, dans ses tentatives de construction de modèles théoriques et aussi, on va y revenir, dans sa propre pratique politique. C'est le schéma de l'écart ou du décalage, qui a un rapport avec la question du centre. La question du centre est, d'un côté, une question spéculative, cosmologico-théologique, et d'autre part, c'est une question politique directement liée au problème du communisme, de la révolution, etc. En effet, le schéma stalinien - et Althusser pense et agit en fonction de ça - c'est le schéma du centralisme, non seulement dans le centralisme démocratique, mais en général il y a le centre politique, le centre théorique du centre politique, qui lui-même est le centre de la transformation sociale, etc. Et Althusser connaissait par cœur les Principes du léninisme de Staline. Alors, je me souviens d'une discussion avec Althusser à propos de la philosophie de l'histoire de Hegel dans laquelle je lui avais dit : « Tu as tort de croire que chez Hegel la dialectique soit complètement centrée, que toutes les manifestations de l'esprit ne soient que des reflets ou des effets de la puissance du centre ». Mais le fait est qu'Althusser s'intéresse vivement au fait que les philosophes "matérialistes" sont des gens qui divisent l'« un » en "deux» ou qui produisent une distance à l'intérieur du centre. Ça devient chez Montesquieu la dialectique de la nature et du principe. Il tenait beaucoup à cette idée. Ce qui est très intéressant est que Hegel s'était servi de ça, avait digéré Montesquieu. Mais Althusser pensait qu'Hegel n'avait pas compris que chez Montesquieu la nature et le principe ne sont pas la même chose et qu'on ne peut jamais les réduire l'un à l'autre. Et la fortune et la virtù chez Machiavel d'une certaine façon constituent aussi une dualité irréductible. On pourrait continuer. Et tout ça est complètement formel, mais c'est une constante.

YD : Là tu touches un point très important : la haine du centre. Et d'une certaine façon François Matheron a mis ça en avant avec la catégorie du vide. La catégorie du vide, c'est justement la distance d'avec le centre. Il y a un écart qui n'est pas réductible. Et ce qu'Althusser pensait de Hegel, c'est que finalement même si les choses partaient très loin elles étaient toujours rattrapées. fondamental qui relevait d'histoires de curé. YD : Cette question de l'écart, de la distance, du vide d'une distance prise, cette phrase qui avait suscité une sorte d'émotion quasi-mystique chez le père Breton...

Andrea Cavazzini: Mais c'est une formule mystique. Il s'agit du décentrage ou du décentrement qui est en réalité une distance minimale, presque inexistante par rapport au centrage. YD : Le décentrement on peut considérer que tu restes lié au centre... Le vide et la distance prise c'est qu'il y a un écart, je le répète, qui n'est pas réductible. La catégorie de vide revient à Lucrèce... AC : À Maître Eckhart aussi...

EB : Absolument, mais Andrea a tout à fait raison, c'est d'un côté Lucrèce et de l'autre la théologie... Alors évidemment savoir comment on peut tenir ensemble les mystiques et le courant souterrain du matérialisme, c'est une grande question. Mais c'est vrai qu'il ne faut pas se contenter du matérialisme, qu'il y a aussi toute la tradition mystique.

AC : À propos de "l'Un qui se divise en Deux», on peut dire que dans une certaine tradition néoplatonicienne en effet l'Un ne consiste que dans sa division... 
EB : Mais Althusser était en même temps très méfiant à l'égard de ça parce qu'il mobilisait une autre formule qui est « diviser pour mieux régner ». Althusser avait transformé ça - à un moment où les maos s'étaient mis à réciter « Un se divise en Deux, Deux fusionnent en Un » comme une prière du matin - en «Un se divise en Deux pour mieux régner »! Avec l'idée que justement chez les néoplatoniciens en particulier, dont il savait quelque chose il savait toujours plus que ce qu'il prétendait, mais moins évidemment qu'il aurait voulu ou qu'on aurait pu espérer -, il ne suffisait pas de le diviser en deux pour se débarrasser de l'Un. C'est pour ça qu'il cherchait tout le temps des suppléments, comme dirait Derrida, pour que la division en deux reste un écart irréductible. Il s'agit par exemple de l'inégalité des origines, de l'idée qu'il faut que les deux soient hétérogènes, qu'ils soient inégaux. On reviendra à la question de la politique, mais plus je réfléchis à tout ça plus je me dis qu'Althusser a essayé, fantasmé d'être politiquement efficace dans la mesure où il se tiendrait à l'écart du centre, mais dans le voisinage immédiat du centre.

51 YD : Il y a aussi la question du rôle qu'a joué la lecture de Gramsci dans le rapport d'Althusser à Machiavel dans les années 60 et 70 . Mais là je suis court, parce que j'ai très peu, presque jamais parlé de Gramsci avec Althusser. Quand je suis entré à l'École normale, mon grand personnage c'était Gramsci. J'en parlais avant même de connaître quoi que ce soit au marxisme. Je trouvais ça extraordinaire. Mais le problème, c'est que après - Étienne dit que c'est stalinien, bon il exagère un peu -, en lisant le texte sur Gramsci dans Lire «Le Capital ", j'étais d'accord parce qu'on ne pouvait pas ne pas être d'accord, mais c'est vrai que je n'en ai pas parlé avec lui.

EB : Moi j'ai eu des discussions sur Gramsci avec Althusser, comme ça entre deux portes, à différentes époques. Parmi les inédits, dans un des textes inachevés de la fin de «Marx dans ses limites ${ }^{30}$, il y a un grand chapitre sur Gramsci. Je me rends compte, et Vittorio me le confirme, qu'Althusser est dans un mouvement d'attraction-répulsion, de balancier permanent. À peine il a écrit un texte pour dire que Gramsci c'est impossible, il en écrit un autre pour dire que c'est le seul qui nous aide à penser. Dans ces années-là, les choses vont très vite. Celui où il se rapproche le plus de Gramsci dans la dernière période, c'est le texte de la conférence de Grenade qui s'appelle «La transformation de la philosophie »31. Là encore, je suis pris entre deux feux, dans des sentiments très contradictoires parce que la revue Période a rendu publique, il y a quelques mois, la conférence de Barcelone sur la dictature du prolétariat ${ }^{32}$. Alors cette histoire, je ne dis pas qu'elle me prend aux tripes, mais enfin elle a une certaine importance pour moi, parce qu'Althusser m'avait fait écrire un livre sur la dictature du prolétariat que lui ne voulait pas ou ne pouvait pas écrire. J'avais fait de mon mieux, un mélange de militant réformiste révolutionnaire, et puis de prof de philo. Ce qui m'amuse quand je relis mon propre texte c'est que je vois qu'au fond j'avais attrapé des machins qui sont devenus de la tarte à la crème à partir du moment où les gens ont lu Carl Schmitt et d'autres sur la dictature, sur l'état d'exception. Je racontais ça avec Lénine, ce qui prouve que c'est chez Lénine d'une certaine façon.

AC : Schmitt ne l'aurait pas nié d'ailleurs...

EB : Mais c'est toujours pareil, je ne savais pas ce que je faisais, et puis là-dessus Althusser fait une conférence quelques mois après. Mais cette conférence me terrifie. Elle me donne le sentiment qu'Althusser est le prisonnier non pas d'une cage d'acier comme dirait Max Weber, mais d'une camisole de force : il sent le communisme se désagréger et il fait un effort à la fois surhumain et dérisoire pour expliquer que le marxisme c'est vrai, le communisme c'est vrai, le léninisme c'est vrai, la dictature du prolétariat c'est le cœur, qu'il ne faut pas céder là-dessus, etc. Bon, ça prouve qu'il y croyait. Mais dans le même 
moment ou presque il fait cette autre conférence à Grenade où il dit : "Il nous faut une théorie de l'hégémonie, j'essaie d'y contribuer avec ma conception du sujet, Gramsci est le seul qui y a compris quelque chose ». Le moins qu'on puisse dire c'est que les deux choses ne vont pas dans le même sens.

YD : Ça nous mène au communisme. Et c'est vrai que dans cette conférence sur la dictature du prolétariat, que je n'ai pas lu parce que ça m'est tombé des mains, mais j'ai regardé un peu quand même la fin, il reprend la phrase célèbre où Marx dit qu'il n'a pas inventé la lutte de classes, car c'est Guizot, etc., mais qu'il a inventé la dictature du prolétariat qui mène au communisme. C'est la lettre à Weydemeyer de 1852 qui est rituellement citée ad nauseam. Concernant le communisme, vous employez le mot d'idée idée communiste -, ça c'est Badiou. Et ça ne peut pas être Althusser parce qu'Althusser n'est jamais allé plus loin sur la question du communisme que la fameuse phrase de l' Idéologie allemande, tout le temps répétée, selon laquelle c'est le mouvement réel qui abolit - Aufhebung - l'état des choses existant. À propos de ce qu'il disait du communisme - c'est une chose qui m’avait toujours frappé - je lui ai demandé plusieurs fois, il ne me répondait jamais : «C'est quoi tes îlots communistes ? Pourquoi le communisme est déjà là d'une certaine façon?» Et là, je dirais, c'est l'inventaire à la Prévert. Il y a quatre choses: 1) la collectivisation de la production par le capitalisme. J'avais expliqué à Althusser - une fois il l'a lui-même dit dans une réponse aux gens du Parti - que des socialisations, il y en avait au moins deux qui n'avaient rien à voir l'une avec l'autre : il y avait la socialisation marchande et la socialisation à l'intérieur de l'entreprise capitaliste. 2) Les syndicats, les organisations. 3) Les mouvements de masse. 4) Peut-être les inventions des chercheurs, des artistes. J'appelle ça un inventaire à la Prévert. Je pense qu'en termes de position subjective, Althusser était communiste. Le problème c'est de comprendre pourquoi il voulait en trouver des réalisations. Il savait trop qu'il ne pouvait pas être utopiste ; il s'appuyait sur cette phrase de Marx qui dit que quand on va tomber il faut s'accrocher quelque part. Du coup il produisait des inventaires à la Prévert. Il n'avait pas travaillé là-dessus, il n'était pas avancé...

EB : D'accord, l'idée du communisme, c'est une formule de Badiou, et je pense en effet qu'elle ne va pas pour Althusser. Tu as dit « subjectivement communiste ». Évidemment Badiou rattache la subjectivité à une idée. Je pense qu'on peut dire que, dans le cas d'Althusser, il faut prendre les choses dans l'autre sens. C'est-à-dire qu'il y a une subjectivité communiste d'Althusser qui précède toute idée. Ce sont des choses qui peuvent toucher d'une part un peu à la mystique - d'autant qu'il y a un fond chrétien chez Althusser, il ne faut pas l'oublier...

YD : Mais c'est pour ça qu'il fallait absolument cette phrase de l'Idéologie allemande.

EB : ...et puis d'autre part il a pris des expériences tout à fait quotidiennes dont on peut se poser la question de savoir si elles sont spécifiquement liées au nom de communisme - il n'y a pas de raison non plus de ne pas les rattacher au nom de communisme - ; il y a des gens qui appelleraient ça fraternité, égalité, de toutes sortes de façons. Ce n'est pas une idée, mais c'est plutôt - je suis désolé d'aller puiser du côté d'une mauvaise philosophie existentiel, de l'ordre du vécu.

YD : C'est pour ça que je dis subjectif. Sauf, tu connais quand même la formule qu'il avait essayé d'inventer; il m'a dit qu'il l'avait trouvée avec Jacques Martin. Il y a une définition du communisme chez Lénine que tout le monde connaît : c'est l'électricité plus les soviets. Et avec Martin ils avaient ajouté une troisième chose: la psychanalyse pour tout le monde ! 
60 AC: D'ailleurs, pour rebondir sur ce qu'Étienne vient d'appeler « une mauvaise philosophie ", c'est intéressant que Sartre disait dans un texte du début des années 50, plus ou moins contemporain des "Communistes et la paix ", plus ou moins la même chose - à savoir que dans les sociétés communistes il y aura une généralisation de la pratique psychanalytique.

61 EB : Mais moi, je pensais plutôt au fait que pour interpréter la formule qui dit qu'il y a des éléments de communisme qui sont déjà là, dans les pores de la société, on peut chercher dans deux types de directions. Ou bien c'est ton inventaire à la Prévert, on peut chercher à identifier des faits sociaux ou sociologiques, éventuellement même des structures ou des microstructures ou des tendances de transformation ou d'innovation structurelle, ou bien au contraire on peut aller - évidemment toute la cuirasse anti-humaniste qu'on s'était fabriquée, qu'Althusser lui-même avait encouragée, même s'il ne la pratiquait pas pour lui-même tout à fait, nous éloignait de ça -, du côté d'une réponse d'un type complètement différent qui est justement existentiel, vécu, expérientiel. Pourquoi y a-t-il déjà du communisme dans les pores de la société ? Le problème véritable est : est-ce que nous pouvons en faire l'expérience ? Spinoza disait « nous sentons et nous expérimentons que nous sommes éternels " et on aurait pu dire qu'il y a des circonstances dans lesquelles nous sentons et nous expérimentons que le communisme est là. À ce momentlà, il se joue une question qui n'est pas tout à fait négligeable et qui a beaucoup à voir avec l'histoire des utopies révolutionnaires, qui est de savoir si ce genre d'expérience ça peut se faire avec n'importe qui. Althusser va dans ce sens dans un des derniers textes auquel j'ai des raisons particulières de m'intéresser parce qu'il me l'avait envoyé et je lui avais dit: «Mais qu'est-ce que c'est que cette horreur!». C'était le texte (inédit) dit des «Thèses de juin », à propos duquel je lui avais dit : « Mais tu retournes à l'Église ! Ce n'est pas possible!». Dans ce texte il disait: «Partout où dans notre société des gens établissent entre eux des relations non marchandes, qui ne sont pas médiées par la marchandise et par la monnaie, il y a du communisme ». Évidemment d'un côté ce qui me frappait, c'est qu'Althusser nous avait toujours dit qu'il fallait laisser de côté la première section du Capital, que le problème fondamental ce n'est pas la marchandise, c'est le mode de production, l'exploitation. Et maintenant il disait, comme tout marxiste humaniste qui se respecte, que si on se débarrasse de la marchandise on est dans le communisme. Mais il $\mathrm{y}$ a un autre aspect frappant de ça. Si on prend ça comme critère - on va faire du mauvais Badiou, ou du Nancy, Blanchot, etc. - les expériences amoureuses sont des expériences communistes. Alors, ou bien il $\mathrm{y}$ a toute sorte de circonstance dans la vie où on se dégage du marchand ou où en tout cas il n'a pas prise sur tout, et donc on s'aime, on se reconnaît, on se comprend même sans parler, on se traite en égaux, enfin toutes sortes de choses très bien, et on est dans le communisme. Ou bien on pense qu'il y a quand même un autre aspect qui est l'aspect ouvriériste d'Althusser, suivant lequel c'est par excellence les ouvriers qui font ce genre d'expérience, que c'est si on rencontre des ouvriers qu'on peut éprouver dans sa forme la plus intense la fraternité communiste, etc.

YD : C'est un aspect, mais quand je dis l'inventaire à la Prévert c'est qu'il y a les ouvriers, mais à côté il y a aussi autre chose - il peut y avoir les étudiants, les mouvements de masse, etc.

EB : Alors il s'agit de savoir d'où vient cet aspect-là. Une grande tentation pour moi et pour d'autres, c'est de dire que ça vient du passé chrétien d'Althusser - et plus précisément catholique. Évidemment le fait que j'ai lu depuis quelque temps des choses qui ont paru, ajoutant à ce que Moulier-Boutang avait expliqué dans son livre ${ }^{33}$, en 
particulier un ouvrage sur Jeunesse de l'Église ${ }^{34}$, la thèse d'un jeune historien lyonnais Lyon évidemment c'est l'épicentre du catholicisme français, c'est là qu'Althusser avait été formé dans sa jeunesse. Ça porte sur l'histoire du mouvement de déviationnistes catholiques auquel Althusser a appartenu tout de suite après la fin de la guerre, dont le gourou était un dominicain qui s'appelle le père Montuclard, avec sa maitresse qui était la mère - c'est un peu comme chez les saint-simoniens - du groupe, et Althusser qui était le fils ainé, le bras droit de ce dominicain, qui avait vu en lui un génie théorique en herbe. Ils se complétaient parfaitement et il a écrit pour le mouvement quelques textes. Alors, à ce moment-là, il est tentant de dire que l'aspect existentiel et érotico-mystique de la conception d'Althusser sur le communisme qui ressort, ça vient de ses expériences de jeunesse, de ce mouvement ou de son langage. Mais en fait c'est un peu abusif de dire ça, parce que c'est quelque chose qui traverse toute l'histoire et qui n'est pas nécessairement catholique ou chrétien.

La deuxième chose que je veux ajouter est que celui-ci est seulement un des côtés de la question du communisme d'Althusser, c'est le côté subjectif. Évidemment aujourd'hui il n'est question que de sujet - on pourrait ne s'intéresser qu'à ça. Ce à quoi les gens ont du mal à s'intéresser, c'est au fait que pour Althusser le communisme, c'est fondamentalement le Parti communiste, c'est l'organisation communiste. Évidemment, à la fin de sa vie, il a fini par déclarer dans un moment de semi-délire qu'il fallait travailler à la réunification des deux grandes organisations révolutionnaires de l'histoire : le Parti communiste et l'Église catholique. Mais même en laissant de côté l'Église catholique et en se concentrant sur le Parti communiste, il est sûr qu'Althusser - et là on revient à la dictature du prolétariat - a eu les plus grandes difficultés à admettre que justement le communisme n'était plus dans son Parti, pourrait ne pas être dans son Parti, si pourri que soit le Parti. C'est quelque chose qui s'est peut-être effondré en 78 - le texte de Venise ${ }^{35}$ de ce point de vue traduit un désarroi pathétique.

YD : Et pourtant il écrit quand même des textes à ce moment-là contre le Parti communiste.

EB : Oui, mais moi je sais comment il les a écrits ces textes, en se forçant lui-même de façon terrible en essayant de trancher le nœud gordien. Mais jusqu'à ce moment-là au moins, non seulement Althusser n'a jamais renoncé à l'idée que le communisme était indissociable du Parti communiste, de l'existence du Parti communiste, mais il a tenu dur comme fer à l'idée que ce qui distinguait le communisme de toute autre aspiration subjective à la fraternité, c'est que c'était un mouvement politique organisé.

YD : Là-dessus chez Badiou, c'est la même chose. Aujourd'hui il ne parle plus de ça, mais quand il parlait de ça il était sans arrêt en train d'expliquer que justement ce qui le distinguait de tous les autres c'est qu'il était pour l'organisation. Évidemment une organisation hors-État, parce que là-dessus tout le monde était d'accord pour dire « horsÉtat », ce qui est quand même court, parce que finalement...

EB : Plus que court, c'est contradictoire!

YD : Oui, je trouve que c'est contradictoire, parce qu'il faut avoir un concept d'État un peu plus précis. C'est pour ça que je ne suis pas complètement d'accord quand vous dites: « $\mathrm{Ce}$ qui rend impossible toute réduction de la politique à un mode de gouvernement », parce que la question c'est le mode de gouvernement.

69 EB : Althusser est très ambivalent à cet égard, mais ça a aussi des racines dans le catholicisme de sa jeunesse, même si je ne vais pas dire qu'Althusser a répété toute sa vie 
l'expérience de jeunesse qu'il avait fait au sein de l'Église. C'est tellement facile, tellement tentant de suggérer que, y compris pour des raisons psychanalytiques, Althusser ne pouvait pas se détacher d'une structure maternelle, que ça avait d'abord été l'Église et qu'ensuite c'était devenu le Parti communiste. C'est de la psychanalyse de trois sous. Mais ceci étant dit, on ne peut pas complètement négliger les analogies structurales. Le rapport d'Althusser à l'Église catholique est vraiment un double rapport au pôle organisationnel qui peut même à la limite s'autonomiser par rapport à la foi ou à la théologie... C'est Maurras, c'est le maurrassisme, c'est ce que dit déjà Auguste Comte. D'ailleurs Maurras était comtien et ça, c'était un point très important de la formation intellectuelle d'Althusser.

EB : Alors, d'un côté, il y a une chose qui éloignait Althusser de tout gauchisme évidemment, au sens de tout spontanéisme : c'était l'idée que la politique se fait dans, avec, par des organisations qui doivent être des organisations antiétatiques - avec toute l'ambiguïté que ça comporte - qui sont un autre État en face de l'État. FB : Alors, c'est toute la question, est-ce qu'il s'agit d'un miroir de l'État?

EB : Oui, et dans ce cas, tu es piégé, tu es coincé. Mais, d'autre part il y a évidemment une tendance communautariste, spontanéiste, anti-organisationnelle qui conduit périodiquement à l'intérieur de l'Église à la formation de dissidences et d'expériences d'autonomie que l'Église naturellement quelques fois utilise, mais le plus souvent finit par supprimer.

FB : J'ai l'impression que pour Althusser l'action politique communiste doit être deux fois hors-État. C'est-à-dire qu'elle est dans le Parti en tant qu'organisation hors-État, mais qui se constitue dans un rapport en miroir avec l'État, mais elle est aussi en décalage par rapport à cette organisation.

EB : C'est là qu'on va retomber sur la question du décalage. Justement c'est cette idée-là que je voudrais essayer de clarifier un petit peu. Au fond, il y a deux façons d'imaginer que le Parti communiste ne soit pas purement et simplement la réplique de l'État, la machine d'État alternative qui attend son heure. Celui-ci est finalement le schéma du double pouvoir qui a joué un rôle tellement important dans l'interprétation de la conjoncture révolutionnaire de 1917 après les textes de Lénine, lesquels d'ailleurs ont été une fois de plus extrapolés et transposés du plan de la tactique sur le plan de la stratégie. Les textes sur la transition transforment facilement ce que Lénine avait dit: «Nous sommes dans une situation avec un double pouvoir, d'un côté il y a le pouvoir du tzar qui est encore là, le vieil appareil d'État, et puis de l'autre il y a un pouvoir des soviets qui existe déjà et qui est en train de défier le premier; cette situation est une situation instable, elle ne peut pas durer et il s'agit de savoir dans quel sens elle va être résolue ». Ceci est transformé - justement je trouvais ça très bien quand j'écrivais mon livre sur la dictature du prolétariat - en l'idée d'une situation d'instabilité de longue durée. Ça allait avec l'État non-État. Je trouvais magnifiques les textes de Lénine sur la NEP - évidemment ceux qui ont été ensuite refoulés à partir du moment où Staline a déclaré qu'il fallait mettre fin à la NEP - parce que c'était l'idée que le socialisme ce n'est pas le pur pouvoir de la classe ouvrière - c'était maoïste aussi un peu -, c'est une longue phase, peut-être interminable, de concurrence entre le pouvoir de la bourgeoisie ou de ce qui la remplaçait et celui des travailleurs avec des avancées, des reculs, des négociations.

Enfin, il y a deux façons de penser que le Parti communiste n'est pas simplement la réplique de l'État. L'une c'est d'expliquer que l'organisation révolutionnaire ne 
fonctionne pas de façon verticale, comme une organisation de pouvoir sur la base, sur les masses, mais que les masses ont une autonomie, un véritable contrôle sur la direction, que c'est elles qui sont en dernière analyse la force décisive, etc.; c'est donc de valoriser la base, ce qui n'est évidemment pas le cas de l'État, si démocratique soit-il, la question étant alors de savoir naturellement comment les masses font pour se servir d'une direction qui ne les domine pas, ou pas entièrement. On peut voir ça de façon institutionnelle ou on peut voir ça de façon événementielle, périodiquement. C'était le schéma qu'on avait cru lire dans les textes de Mao sur la révolution culturelle. Naturellement, c'est la nature du Parti de se transformer en un autre appareil d'État qui domine les masses, surtout s'il est au pouvoir. Mais périodiquement, les masses vont se révolter ou elles vont se faire entendre, et la direction du Parti sera obligée de reconnaitre que le maitre c'est le peuple, etc.

L'autre possibilité - je crois qu'Althusser dans sa période théoriciste a pensé à ça, ensuite il y a renoncé - c'est ce que j'appelle la "potestas indirecta », c'est le décalage intellectuel. Il pense que ce qui fait du Parti un appareil d'État, comme il l'explique à nouveau dans le texte de la conférence de Grenade, c'est le fait que l'unité du Parti est cimentée par une idéologie, par une théorie, qui est elle-même une idéologie d'État. On peut se poser la question de savoir si c'est l'État qui produit l'idéologie pour assujettir les citoyens ou bien si l'idéologie se produit encore au-delà et fait de l'État l'instrument de sa mise en œuvre, mais de toute façon il y a un emboitement ou une coïncidence de la fonction idéologique et de la fonction administrative ou gouvernementale. Alors, Althusser, je crois, avait tiré de la période stalinienne, qu'il avait vécue, non pas une hostilité de principe à l'organisation de type bolchevique - bien au contraire je pense qu'il croyait tout à fait, en tout cas pendant toute la première période au moins jusqu'à 68 , sinon même encore après, qu'il n'y a pas de révolution sans un Parti révolutionnaire centralisé -, mais il pensait que le Parti ne resterait révolutionnaire que s'il existait une fonction théorique qui lui soit à la fois interne et non subordonnée. Évidemment, dans la pratique ça conduit à tous les compromis les plus boiteux ; c'est pour ça qu'il écrit une lettre à Krasucki pour lui dire: "Je vous offre mes services». C'est une façon très étrange de lui offrir ses services et ceux de ses disciples. Ça consiste à dire : « Nous nous mettons au service de la politique du Parti pour la reformer et l'améliorer, à condition que dans le même temps vous reconnaissiez que nous n'en sommes pas les courroies de transmission». Alors évidemment tout le monde pousse aussitôt les hauts cris, aussi bien les gauchistes que les dirigeants du Parti: "Qu'est-ce que c'est que cette prétention faramineuse des théoriciens de juger par eux-mêmes, de théoriser par eux-mêmes; ils veulent se transformer en nouveaux philosophes rois ! Cette tentation existe évidemment. Je crois qu'il a beaucoup travaillé là-dessus. J'appelle ça la potestas indirecta parce que dans le chapitre du Léviathan qui était ensuite l'une des sources fondamentales d'Auguste Comte sur le pouvoir spirituel, Hobbes avait repris au cardinal Bellarmin cette formule qui consistait à dire : «Certes l'Église n'est plus l'armature du pouvoir politique comme dans l'empire du Moyen-Âge, mais elle demeure la source du pouvoir spirituel qui doit pouvoir s'exercer partout, etc. »

Évidemment, ce qui est encore plus tentant c'est de rassembler les deux, en disant qu'au fond ce qui distingue le Parti révolutionnaire d'un appareil d'État, c'est à la fois le rapport non vertical qu'il entretient avec les masses, à supposer que ça soit possible - toutes les expériences dissidentes communistes de cette époque, surtout en Italie encore plus qu'en France, ont cherché à faire exister cette intervention autonome des masses qui apprend en quelque sorte au Parti lui-même quelle doit être sa politique, ou qui le contraint, ou 
qui le remplace -, et d'autre part l'idée de l'autonomie relative de la fonction théorique qui est à la fois proche du centre, en vue du centre, et néanmoins irréductiblement distincte de façon à ne pas pouvoir devenir son valet, sa courroie de transmission. Évidemment, ce qui est rigolo est que si tu prends les deux ensemble tu retrouves, peutêtre pas par hasard - d'ailleurs c'est en rapport avec les idéologies romantiques prépositivistes -, l'idée du jeune Marx dans les textes de 1844, que je crois pouvoir lire encore d'une certaine façon dans la préface à la Contribution pour une critique de l'économie politique de 1859, selon laquelle pour qu'il y ait du mouvement révolutionnaire - on serait tenté de dire : pour que le Parti politique soit l'agent ou l'instrument d'un mouvement révolutionnaire -, il faut une espèce d'alliance entre la fonction théorique ou philosophique comme disait Marx à l'époque, d'un côté, et puis la puissance autonome des travailleurs ou des prolétaires. Évidemment, on tourne toujours en rond, c'est-à-dire qu'on se demande ce que serait un Parti communiste qui, d'une part, ferait place à la fonction théorique comme telle, laquelle n'est pas la fonction de fournir des arguments pour justifier la ligne du Parti, et d'autre part, conférerait en son propre sein aux masses qu'il représente une fonction de contrôle et peut-être même de direction. Alors là, on voit bien qu'évidemment le stalinisme constituait l'anti-modèle parfait parce qu'il avait réduit les deux, mais personne n'a jamais trouvé, que je sache, la pierre philosophale.

FB : Oui, et la question se pose de savoir si l'organisation dans ce schéma reste du côté du Parti en tant qu'il tend à devenir appareil d'État et non pas de deux autres côtés, ou si on ne la retrouve pas de l'autre côté aussi.

EB : Je crois qu'on pourrait dire que ce qu'Althusser pensait, peut-être Yves a raison de dire que Badiou et d'autres aussi, et en Italie je suis sûr qu'on trouverait des équivalents, est que justement l'organisation, c'est-à-dire le quasi-État ou l'anti-État est la matérialité, la condition. S'il n'y a pas de Parti, s'il n'y a pas d'organisation, les philosophes d'un côté et la spontanéité des masses de l'autre ne se rencontrent jamais, flottent dans l'air. Le Parti c'est ce qui les réunit, au risque de les asservir.

FB : À propos de communisme, j'ai une question qui est peut-être un peu étrange : est-ce qu'il y avait quelque chose chez Althusser, du moins dans les années 60 , qui relevait de ce qu'on pourrait appeler un « communisme des esprits» suivant l'expression d'Alexandre Matheron référée à Spinoza ${ }^{36}$ L'idée d'une élévation qui va avec une désubjectivation par la théorie.

EB : C'est possible. Alors ça, c'est typiquement le genre de choses dont il ne nous aurait pas parlé à nous, parce que ce n'était pas ça qu'on lui demandait. Je ne dis pas qu'Althusser nous disait juste ce qu'on avait envie d'entendre, mais il avait quand même un discours très adapté - ça aussi c'est très catholique - à la psychologie et à la condition sociale et institutionnelle de chaque interlocuteur.

79 AC : Mais, Yves, je me souviens que tu m'avais dit, lorsqu'on parlait de la centralité de Malebranche dans la pensée d'Althusser, que la différence, la rupture idéologie/science chez Althusser vient en fait de Malebranche, qu'elle a des analogies avec la vision des choses en Dieu qui permet de situer l'idéologie, la vision «fausse ». Et cela me semble aller un peu dans la direction de cette hypothèse que chez Althusser on pourrait trouver une idée du communisme des esprits, un communisme de la vision intellectuelle des choses. 

chose à distance. communisme en contestant la pertinence du vocabulaire badiousien à propos de l'Idée du communisme. Puis on a fini par dire, d'une manière un peu perverse, que chez Althusser il y a le communisme des esprits, et donc la convergence des esprits et leur égalité dans l'Idée et qu'il y a même l'Idée d'un communisme pensé sur le modèle de l'Église : d'un côté la hiérarchie, de l'autre la fraternité communautaire, certes, mais il s'agit dans l'un comme dans l'autre cas de collectifs dominés précisément par une Idée. Et Étienne parlait tout à l'heure de l'autonomie, voire parfois d'un certain primat par la distance ou par la prise de distance, de la fonction idéologique ou philosophique; donc finalement si le communisme n'est pas une Idée, il y a tout de même des idées communistes, ou une fonction idéale ou idéative du communisme.

EB : Mon objectif n'est pas à tout prix de prendre le contre-pied de ce que dit Badiou, ça serait complètement ridicule. En plus sur la question de l'idée communiste, pour des raisons en partie rhétoriques et circonstancielles, mais pas totalement, quand on a discuté à New York, je lui ai dit : «Tu as raison, sauf que moi je n'en tire pas tout à fait les mêmes conséquences que toi ". Je n'ai dès lors pas de raison de vouloir échapper à ça à tout prix. Ceci étant dit, il n'est pas interdit de faire un petit effort philosophique supplémentaire, surtout après avoir cité des références aussi diverses que le platonisme d'un côté, l'interprétation par Matheron du troisième genre de connaissance et du livre $\mathrm{V}$ de l'Éthique, et d'autres choses encore. Faire une lecture platonicienne de la cinquième partie de l'Éthique, ce n'est pas du tout impossible... d'ailleurs ça été fait. Mais on peut aussi se demander, comment relancer la question de la différence entre une communauté intellectuelle de participation à une idée ou à un idéal et une communauté de travail théorique qui soit cimentée par une idéologie, par une idéologie commune. Le concept althussérien d'idéologie était quand même très profondément spinoziste et il était fondé sur une lecture un peu particulière de Spinoza, que je ne suis pas éloigné de penser vraie, selon laquelle on ne sort pas du premier genre de connaissance, ce qui ne signifie pas que ce soit le seul : toute expérience intellectuelle, toute production d'idées se perçoit ellemême, et dans la mesure où elle se perçoit elle-même, où elle est consciente d'elle-même et se communique - ça se passe dans le premier genre de connaissance. C'est des affects et de l'imagination.

YD : Je dis une dernière chose et puis on termine ce point. En 1966, j'avais passé quinze jours avec Althusser, on avait beaucoup discuté, et il m'avait expliqué qu'après Lire «Le Capital », il allait faire un séminaire sur Spinoza en 67. Il m'a dit: "Qu'est-ce que tu vas faire toi ?»

EB : Ah un séminaire collectif?

YD : Oui comme Lire «Le Capital», alors moi je lui ai dit que j'allais faire: «Pourquoi l'esprit de Parti, c'est la même chose que l'amour intellectuel de Dieu ». Et il m'avait dit: « Vas-y ». Et puis il n'y a jamais eu de séminaire, comme tu le sais.

EB : C'est marrant que tu dises ça parce qu'au mois de mai prochain, notre ami Filippo Del Lucchese a organisé un colloque de deux jours ici à Paris en l'honneur de Pierre Macherey pour l'anniversaire de son livre Hegel ou Spinoza ${ }^{37}$. Je suis bien décidé à y participer, et comme on se trouve dans l'année du cinquantenaire qui fait notre discussion aujourd'hui, 
mes idées en reviennent tout le temps au même point: d'une part je pense que Pierre Macherey, en écrivant ce livre-là, a exécuté après coup des intentions qui étaient sousjacentes à notre entreprise théorique de l'époque de Lire «Le Capital ». Il l'a exécuté après coup. Naturellement en ayant fait des cours sur l'Éthique. Ce n'est donc pas complètement aberrant de rattacher ça à la conjoncture intellectuelle dont nous parlons. D'autre part, dans la préface de Lire "Le Capital ", Althusser a mis une petite note pour renvoyer à un article de Pierre Macherey, "À propos de la rupture», pour justifier la reprise de la formule de Spinoza "verum index sui et falsi », comme formulation spinoziste si j'ose dire de la théorie marxiste de l'idéologie.

YD : En insistant sur le «et ».

EB : En insistant sur le "et ", oui. Je dis toujours à Pierre : "Tu as fourni à Althusser une pièce de son dispositif ». Alors naturellement, c'est comme toutes les pièces du dispositif d'Althusser qu'on lui avait fourni, ça venait de lui d'une certaine façon, mais c'était passé par le détour d'une interprétation, d'une élaboration supplémentaire. Et tout ça est entièrement lié à la question du modèle Spinoza.

\section{NOTES}

1. É. Balibar, "L'objet d'Althusser ", in S. Lazarus (éd.), Politique et philosophie dans l'œuvre d'Althusser, Paris, P.U.F., 1993.

2. E. Balibar, Sur la dictature du prolétariat, Paris, Maspero, 1976.

3. A. Badiou, Abrégé de métapolitique, Paris, Seuil, 1998.

4. L. Althusser, L'avenir dure longtemps, Paris, Stock/IMEC, 2007 (1992), p. 493.

5. L. Althusser, « Le "Piccolo", Bertolazzi et Brecht » (1962), in Pour Marx, Paris, Maspero, 1965.

6. L. Althusser, «Lettre à D... $\left(\mathrm{n}^{\circ} 2\right) »(1966)$, in Écrits sur la psychanalyse: Freud et Lacan, Paris, Stock/IMEC, 1996, p. 108.

7. G. Deleuze, Spinoza et le problème de l'expression, Paris, Minuit, 1968.

8. L. Althusser, E. Balibar, R. Establet, P. Macherey, J. Rancière, Lire «Le Capital» (2 vol., Paris, Maspero, 1965), Paris, PUF, 1996.

9. " Jacques Rancière : "C'était un enchanteur" ", Le Magazine Littéraire, n 551, février 2015.

10. L. Althusser, Pour Marx, Paris, Maspero, 1965.

11. L. Althusser, "L'objet du "Capital” ", in Lire Le Capital, op. cit.

12. L. Althusser, « Du "Capital” à la philosophie de Marx", in Lire Le Capital, op. cit.

13. J.-A. Miller, « Action de la structure », Cahiers pour l'Analyse, vol. 9, été 1968.

14. "Strong Structuralism, Weak Subject: An Inteview with Yves Duroux», in P. Hallward, K. Peden, Concept and Form, vol. II: Interviews and Essays on the Cahiers pour l'Analyse, London-New York, Verso, 2012.

15. J. Rancière, «Le concept de critique et la critique de l'économie politique des "Manuscrits de 1844" au "Capital" ", in Lire Le Capital, op. cit.

16. R. Establet, « Présentation du plan du "Capital” » in Lire Le Capital, op. cit.

17. P. Macherey, «A propos du processus d'exposition du "Capital" » in Lire Le Capital, op. cit.

18. "Étienne Balibar: "Il avait un talent pour instaurer un climat d'égalité" ", Le Magazine Littéraire, op. cit. 
19. V. Morfino, «Lire Gramsci après Althusser ", in Décalages, 1, 1, 2012, http://scholar.oxy.edu/ cgi/viewcontent.cgi?article=1032\&context=decalages.

20. M. Harnecker, Los conceptos elementales del materialismo histórico, Siglo XXI, 1e éd. 1968.

21. L. Althusser, "Matérialisme historique et matérialisme dialectique", Cahiers marxistesléninistes, $\mathrm{n}^{\circ} 11,1966$.

22. L. Althusser, «Sur la politique du Parti à l'égard des travailleurs intellectuels », Nouvelles Fondations, $\mathrm{n}^{\circ}$ 3-4, 2006.

23. L. Althusser, Montesquieu. La politique et l'histoire, Paris, PUF, 1959.

24. L. Althusser, «Le courant souterrain du matérialisme aléatoire» (1982), in Écrits philosophiques et politiques, Tome I, Paris, Stock/IMEC, 1994.

25. L. Althusser, «Contradiction et surdétermination », Pour Marx, op. cit.

26. L. Althusser, «Idéologie et appareils idéologiques d'État », La Pensée, nº 151, juin 1970.

27. M. Foucault, La société punitive. Cours au Collège de France (1972-1973), Pairs, EHESS-GallimardSeuil, 2013.

28. B. Porchnev, Les Soulèvements populaires en France de 1623 à 1648, Paris, SEVPEN, 1963.

29. L. Althusser, « Machiavel et nous ", in Écrits philosophiques et politiques, Tome II, Paris, Stock/ IMEC, 1995.

30. L. Althusser, « Marx dans ses limites », in Écrits philosophiques et politiques, Tome I, op. cit.

31. L. Althusser, «La transformation de la philosophie. Conférence de Grenade (1976) », in Sur la philosophie, Paris, Gallimard, 1994.

32. L. Althusser, "Conférence sur la dictature du prolétariat à Barcelone ", Période, http:// revueperiode.net/un-texte-inedit-de-louis-althusser-conference-sur-la-dictature-du-proletariata-barcelone/

33. Y. Moulier-Boutang, Althusser. Une biographie, Tome I, Paris, Grasset, 1992.

34. Thierry Keck, Jeunesse de l'Église (1936-1955). Aux sources de la crise progressiste en France, Paris, Karthala, 2014.

35. Cf. L. Althusser, « Enfin la crise du marxisme !» (1977), in Solitude de Machiavel et autres textes, Paris, P.U.F., 1998.

36. A. Matheron, Individu et communauté chez Spinoza, Paris, Minuit, 1969.

37. P. Macherey, Hegel ou Spinoza, Paris, Maspero, 1977.

\section{RÉSUMÉS}

Dans cette conversation Étienne Balibar et Yves Duroux interrogent certaines des dimensions les plus actuelles et problématiques de l'œuvre de Louis Althusser. Dans un premier temps, ils reviennent sur la composition de Lire «Le Capital» en abordant la question du rapport entre structure et conjoncture. A partir de là, ils explorent les aspects de la relation d'Althusser avec le communisme, à la fois du point de vue «subjectif » et de celui du rapport avec le PCF. Dans un second moment, ils questionnent le rapport entre science et idéologie ainsi que l'intervention intellectuelle d'Althusser en se penchant tout particulièrement sur son spinozisme et sur son rapport à la psychanalyse. 


\section{INDEX}

Thèmes : psychanalyse, philosophie sociale, philosophie politique, épistémologie, philosophie française contemporaine, matérialisme, structuralisme, marxisme

Index chronologique : années soixante, années soixante-dix, années quatre-vingt Index géographique : France

Mots-clés : Althusser, structure, conjoncture, communisme, dictature du prolétariat, parti, église, science, idéologie, philosophie, Spinoza

\section{AUTEURS}

\section{ÉTIENNE BALIBAR}

Étienne Balibar est professeur émérite de l'Université de Paris-Ouest Nanterre La Défense ; il enseigne à l'Université de Californie à Irvine et à l'Université Columbia.

\section{YVES DUROUX}

Yves Duroux a enseigné l'épistémologie des sciences sociales au nouveau département de sociologie de l'Université de Paris VIII (Vincennes) à partir de 1967. Il a été Vice-Directeur du département des sciences humaines et sociales du CNRS. Il a ensuite enseigné à l'Ecole Normale Supérieure de Cachan de 1993 à 2006, année où il se retire de l'enseignement.

\section{FABIO BRUSCHI}

Fabio Bruschi est assistant et doctorant à l'Université catholique de Louvain où il mène une recherche sur les rapports entre idéologie, action collective et intervention intellectuelle à partir de la pensée de Louis Althusser.

\section{EVA MANCUSO}

Eva Mancuso est aspirante FRS-FNRS attachée à l'UR MAP de l'Université de Liège. Elle mène actuellement une thèse de doctorat intitulée : « Cl. Lefort et L. Althusser : lecteurs de Machiavel ». 